\title{
Evidence for caudate nucleus involvement in an egocentric spatial task: Return from passive transport
}

\author{
L. ABRAHAM \\ University of Texas, Austin, Texas \\ M. POTEGAL \\ New York State Psychiatric Institute, New York, New York \\ and \\ S. MILLER \\ Teachers College, Columbia University, New York, New York
}

\begin{abstract}
Potegal (1972) proposed that the caudate nucleus was involved in processing sensory (especially vestibular) cues for egocentric spatial orientation. The purpose of these experiments was to determine the effect of caudate nucleus lesions on a return from passive transport (RPT) task, which has been shown to depend on vestibular input (Miller, Barnett, \& Potegal, Note 1). In the first experiment, 18 male rats were trained on RPT. Six then received bilateral posterior caudate lesions, 6 received bilateral control lesions in the dorsal hippocampus, and 6 served as sham-operated controls. Postsurgical retraining revealed deficits in RPT for the caudate group, relative to hippocampal and sham groups. To test the specificity of the caudate lesion effect, a second experiment examined the effect of caudate and hippocampal lesions on an exteroceptive-cue-based spatial task, olfactory trail finding (OTF). Neither posterior caudate lesions nor dorsal hippocampal lesions produced deficits in OTF. These results suggest that the role of the posterior caudate in spatially oriented behavior is restricted to egocentric orientation based on vestibular cues. Since the caudate lesions had no effect on air-righting behavior, any vestibulostriatal contribution to RPT must be separate from the regulation of righting reflexes. This contribution may be mediated via the posterior portion of the caudate. Failure to find an effect of hippocampal lesions on RPT supports the suggestion that hippocampal involvement in spatial behavior may be restricted to memory and mapping of exteroceptive cues (O’Keefe \& Nadel, 1978).
\end{abstract}

Investigations of animal behavior following lesions of the caudate nucleus have revealed deficits in performance on spatial tasks involving passive avoidance (Winocur, 1974), alternation (Gross, Chorover, \& Cohen, 1965), and position discrimination (Potegal, 1969). Although these investigations have yielded different measures of performance following somewhat different placement of lesions, together they suggest that the caudate nucleus may be involved in spatial orientation. The purpose of the following experiments was to examine this involvement more closely. Of particular concern in studies of spatial orientation is the nature of the sensory cues that are utilized. One relevant distinction that can be made is whether per-

We thank Susan Hudman, John Blankenship, and Lance Osborne for their assistance in collecting the data. This work was supported in part by a grant from the University Research Institute, University of Texas at Austin. Address reprint requests to: Lawrence Abraham, Department of Physical and Health Education, University of Texas, Austin, Texas 78712. formance is oriented with respect to the environment or the self. Orientation to the environment is based primarily on exteroceptive cues, whereas proprioceptive or kinesthetic cues would be most useful in maintaining egocentric orientation. Although it is obvious that many behaviors can use both types of cues, there is some evidence supporting a distinction between spatial tasks based on available cues (e.g., Juurmaa \& Suonio, 1975; Potegal, 1969; see also O'Keefe \& Nadel, 1978).

Potegal (1972) has suggested that the role of the caudate nucleus in spatial orientation seems to be most evident for egocentric orientation tasks. Several studies have shown that caudate lesions affect spatial tasks only when exteroceptive cues are not available (Douglas, 1966; Mikulas, 1966; Potegal, 1969). Also, patients with Huntington's disease have been shown to exhibit spatial-motor deficits on egocentric tasks (Potegal, 1971).

The return from passive transport (RPT) task described by Miller, Potegal, and Abraham (1983) 
clearly involved egocentric orientation. Hence, it is reasonable to suppose that the caudate might mediate performance on this task. Furthermore, there is a body of evidence suggesting some relationship between striatal and vestibular function (Copack, Dafny, \& Gilman, 1972; Potegal, Copack, de Jong, Krauthamer, \& Gilman, 1971; Spiegel, Szekely, \& Gildenberg, 1965). The dependence of RPT performance on vestibular input (as demonstrated by Miller, Potegal, \& Abraham, 1983) thus also suggests a role for the caudate nucleus in this task.

The first experiment reported here was a test of the effects of bilateral posterior caudate nucleus lesions on RPT performance. Posterior lesion sites were chosen because of (1) evidence of particular involvement in egocentric spatial tasks (Potegal, 1969), and (2) evidence of vestibular input to this region via the magnocellular medial geniculate body (Copack et al., 1972; Ryugo \& Killackey, 1974). Interpretation of the finding of this first experiment that posterior caudate lesions disrupt RPT performance required distinguishing between deficits resulting from specific cue utilization or strategy impairments and more general deficits. Therefore, the second experiment reported here examined the effects of similar caudate lesions on a similarly structured, but nonegocentric, spatial task. In both experiments, control lesions were placed in the dorsal hippocampus, which has been strongly implicated in exteroceptive spatial orientation (Freeman, 1978; O'Keefe \& Dostrovsky, 1971; O'Keefe \& Nadel, 1978; Olton, 1977). In particular, Jarrard (1978) and O'Keefe and Nadel (1978) have argued that the dorsal hippocampus is important in learning complex spatial tasks based on exteroceptive cues.

\section{EXPERIMENT 1}

The purpose of this experiment was to examine the effects of posterior caudate nucleus lesions on reacquisition of an egocentric spatial orientation task (RPT). Performance was measured over successive increments in task difficulty to allow assessment of the degree of any impairment.

\section{Method}

Animals. Eighteen naive male Long-Evans rats (Blue Spruce Farms) weighing 300-325 g were used. The animals were housed individually with ad-lib food throughout the experiment; during training periods, all animals were placed on 23-h 40-min waterdeprivation schedules.

RPT. The experimental task, which previously has been explained in detail (Miller et al., 1983), was to return to a starting point (goal) after passive transport away from the goal in an opaque, wheeled cart. The testing was performed in an eightsided chamber with no salient visual orientational cues except drinking spouts (Mini-LIXIT-25, Systems Engineering Co.) located at each corner (see Figure 1). Thus, the animal, when re-

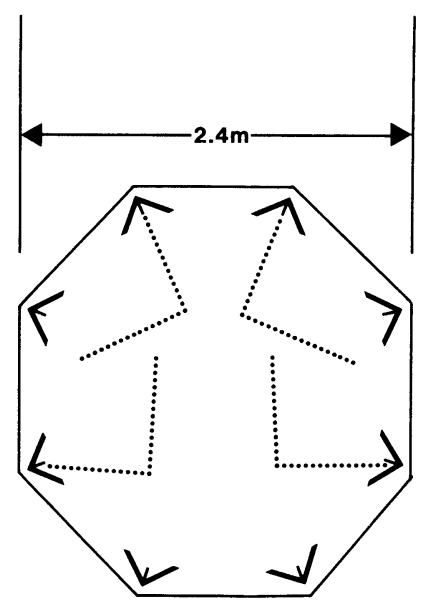

Figure 1. Diagram of chamber arranged for RPT testing. Four sample paths are indicated by dotted lines (path length $=110 \mathrm{~cm}$ ).

leased, was forced to choose, on the basis of the passive transport cues, the correct spout from as many as eight possibilities.

Training. All animals were trained by a titration-shaping procedure to return to the correct water spout over successively more difficult routes. Each daily session consisted of 10 trials. A trial was considered successful if the rat reached the correct water spout within $30 \mathrm{sec}$ without deviating from the target path by more than $45 \mathrm{deg}$. Successful performance on three consecutive attempts at any path length constituted "achieving criterion" for that length and increased the target-path length for the animal by $11 \mathrm{~cm}$. Errors resulted in reduction of the path length for the next trial. For each trial, a map showing the target path and the actual path taken by the rat was constructed. Initial path length was $44 \mathrm{~cm}$, and a maximum performance was set at $110 \mathrm{~cm}$. After 9 days of training, all 18 animals had achieved criterion at $110 \mathrm{~cm}$.

Surgery. The animals were divided into three groups of six, matched on presurgical RPT performance. One group received bilateral lesions of the posterior portion of the caudate nucleus, another received bilateral control lesions of the dorsal hippocampus, and the third group served as sham-operated controls.

Aseptic surgery was performed under sodium pentobarbital anesthesia (Nembutal, $50 \mathrm{mg} / \mathrm{kg})$. Bilateral caudate $(P=1.0 \mathrm{~mm}$, $\mathrm{L}=4.5 \mathrm{~mm}, \mathrm{D}=5.0 \mathrm{~mm})$ and hippocampal $(P=4.0 \mathrm{~mm}, \mathrm{~L}=$ $4.5 \mathrm{~mm}, \mathrm{D}=3.0 \mathrm{~mm}$ ) lesions were made using a stainless steel electrode $(0.25-\mathrm{mm}$ diameter; uninsulated tip $=0.5 \mathrm{~mm}$ long) and a radiofrequency lesion maker (Grass RF-4). The burr holes were then filled with sterile Gelfoam and the scalp wounds closed.

A 10-day recovery period followed the surgery, during which the animals were allowed ad-lib access to food and water.

Retraining. After the recovery period, training was reinstated under conditions exactly duplicating the preoperative training. Initial path length was reset at $44 \mathrm{~cm}$ and increased following three consecutive successful trials as before.

Behavioral evaluation. The performance maps were examined for indications of left-right bias and/or alternation. Daily percent-correct scores were used as an indication of performance independent of path length. Effects of surgical intervention were then examined by subtracting presurgery-achieved path-length scores from postsurgery-achieved path-length scores to obtain savings scores. These savings scores, accumulated over days, proved to be the most sensitive measure of the effects of the lesions on task behavior.

Evaluation of vestibular righting reflexes. Previous work has indicated that air righting in rats is sensitive to disruptions of normal vestibular function (Potegal, Day, \& Abraham, 1977; 
Miller, Barnett, \& Potegal, Note 1; Abraham \& Blankenship, unpublished observations). In particular, head attitude upon landing has been shown to be affected by loss of vestibular (presumably otolith organ) input. Therefore, all experimental rats were filmed using high-speed cinematography (100 fps; Kodak 4-X reversal film) while being dropped from a supine position. Angles of head pitch and roll were determined from the frame of contact using a graphics digitizer (Numonics 1224) interfaced with a laboratory minicomputer (PDP 11/03).

Histology. Upon completion of all testing, the animals were sacrificed with an overdose of Nembutal and the brains perfused with $10 \%$ Formalin. Blocks were cut and fixed in paraffin and then cut in $50-\mu$ sections. Alternate sections were stained using cresyl violet and luxol fast blue techniques. Lesions were evaluated for size and encroachment on surrounding structures.

\section{Results}

Histology. The caudate lesions were located in the ventral portion of the posterior caudate and had a maximum diameter of about $2.0 \mathrm{~mm}$ (Figure $2 \mathrm{~A}$ ). Four of the 12 lesions extended laterally into the overlying cerebral cortex; 3 lesions extended medially into the internal capsule to the lateral border of the thalamus. The hippocampal lesions were found to be entirely within the dorsolateral hippocampus, with a maximum diameter of about $2.0 \mathrm{~mm}$ (see Figure 2B). There were no apparent relationships between lesion size or placement and behavioral data.

General performance. One concern in this type of study is possible motivational effects of a particular lesion that might impair performance. Kirkby (1973) and Schiff and Carter (1977) found that anterior caudate lesions did affect general arousal and food and water consumption. However, Neill and Linn (1975) and Studelska and Beatty (1978) reported only transient aphagia and adipsia following posterior caudate lesions, and Stoller (1974) reported no effects on food or water intake. O'Keefe and Nadel's literature review (1978, p. 471) supported Jarrard's (1976) report of no hippocampal deficits in eating or drinking behavior.

One indicator of motivation deficits may be rates at which animals refuse to run. In these experiments, refusal to run was defined as failure to move to a spout within $30 \mathrm{sec}$ of release. Comparison with preoperative rates revealed no postoperative increases for any of the groups.

Savings scores. Each group showed progress throughout retraining. However, the hippocampal and sham groups showed consistently positive savings scores, whereas the caudate group exhibited only negative savings scores (Figure 3 ). Individual comparisons using the Kruskal-Wallis rank-sum test showed that the caudate group was significantly different from the hippocampal group $(H=13.5$, $\mathrm{p}<.01$ ), but the hippocampal group was not significantly different from the sham group $(H=4.70$, $\mathrm{p}>$.05).

Righting reflex drop scores. Head pitch and roll angles were plotted according to the techniques of
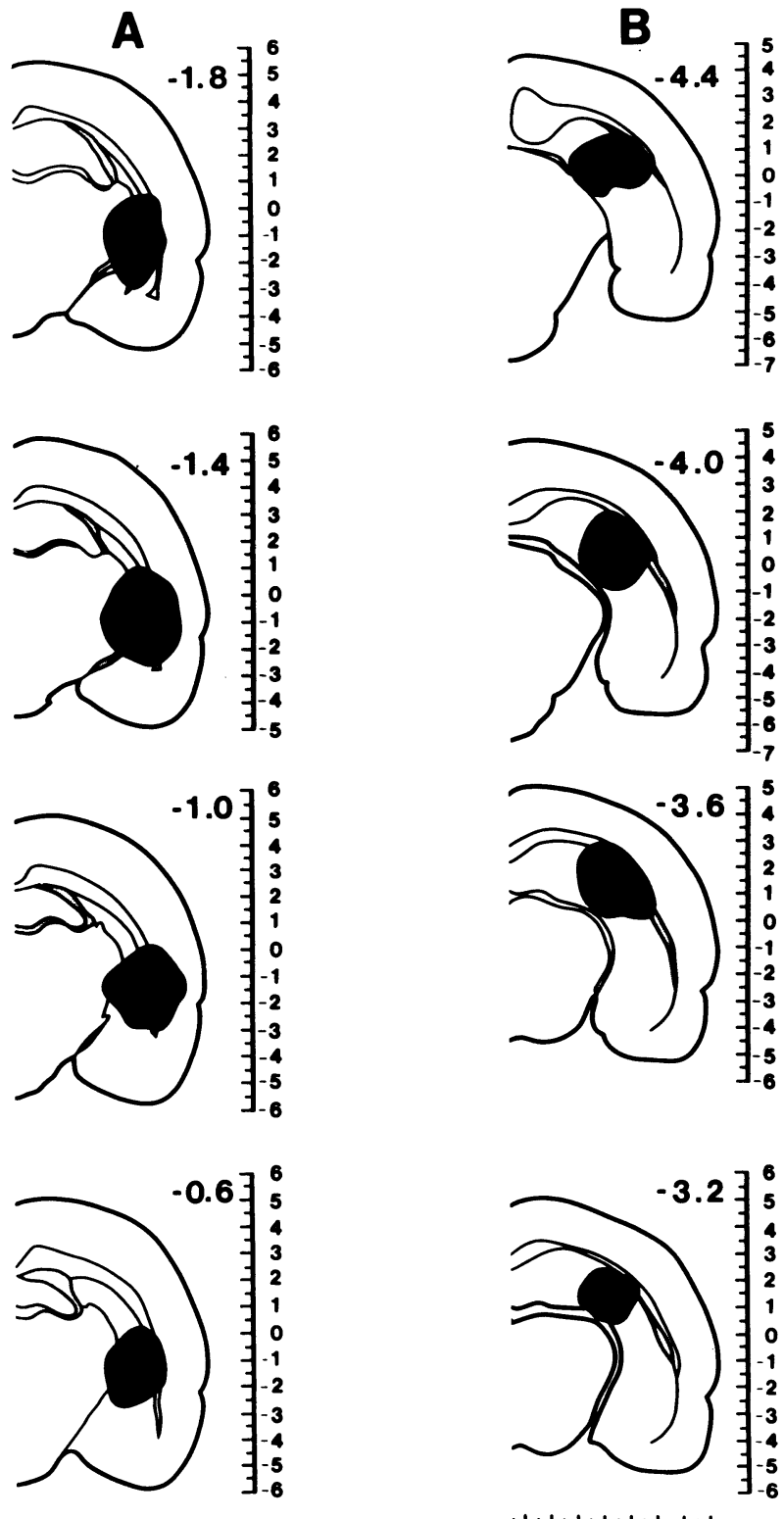

12345678

12345678

Figure 2. Greatest lesion extents at selected coronal sections for (A) posterior caudate lesions and (B) dorsal hippocampal lesions.

Potegal, Day, and Abraham (1977) and are shown in Figure 4. No significant differences were found between groups. This suggests that the head-orientation portion of the righting reflex is not sensitive to bilateral lesions of the caudate nucleus or the hippocampus.

\section{EXPERIMENT 2}

Miller, Potegal, and Abraham (1983) showed that disruption of vestibular function profoundly affected performance on RPT and only transiently 


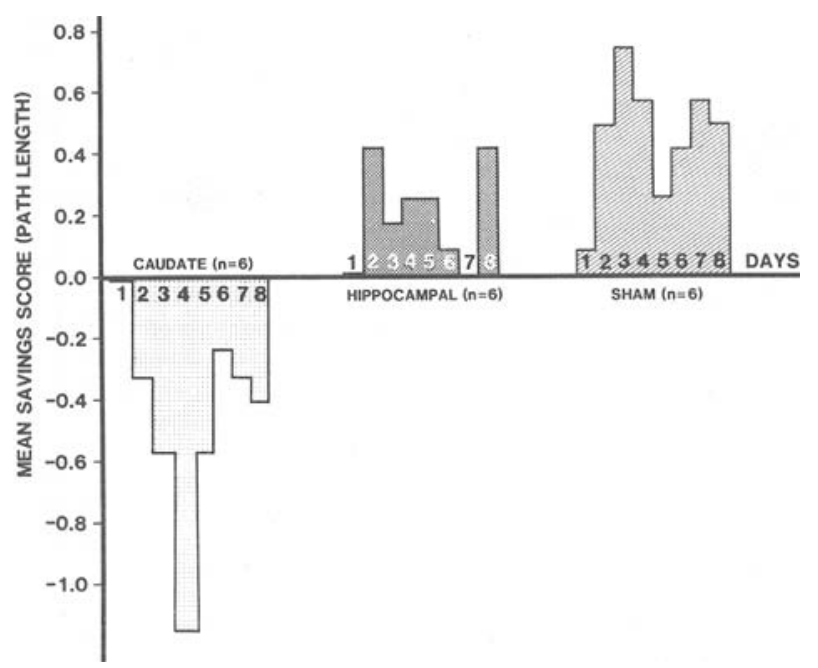

Figure 3. Experiment 1: RPT mean savings scores by days.
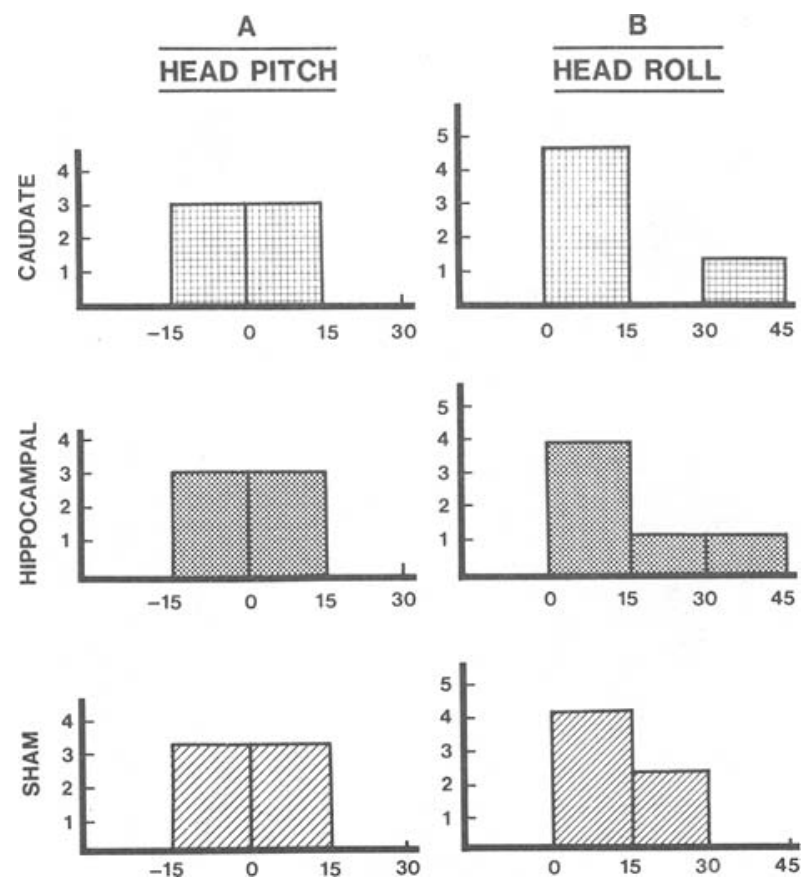

Figure 4. Frequency histograms showing angles of (A) head pitch and (B) head roll from horizontal at moment of landing following drop from supine position.

affected performance on a control spatial task, olfactory trail finding (OTF). To investigate the task specificity of the effect of posterior caudate nucleus lesions, an OTF task involving a choice between eight alternative targets was used with caudate- and hippocampal-lesioned animals. Pilot efforts to train animals on an eight-choice OTF task using water reinforcement were unsuccessful. However, we found that if we used food reinforcement, the animals readily learned OTF.

\section{Method}

Animals. Twelve naive male Long-Evans rats (Blue Spruce Farms) weighing 400-600 g were used. The animals were housed individually with ad-lib water throughout the experiment; during training periods, all animals were placed on a restricted diet of $5 \mathrm{~g}$ of standard rat chow per day. This allowed food to serve as a primary reinforcer for training and performance.

Apparatus and Procedure. As in the OTF task used by Miller, Potegal, and Abraham (1983), the task was to follow a lemonscented string suspended $1 \mathrm{~cm}$ above the floor to a target spot that contained a small chocolate chip. The testing was performed in the same eight-sided chamber used for Experiment 1; the starting point was invariant, and the target path led to a decision point in the center of the chamber $(55 \mathrm{~cm}$ away) from which eight identical strings radiated (see Figure 5). Each string led to a target spot $(55 \mathrm{~cm}$ away) that consisted of a small metal shield obscuring the chocolate chip from sight. Thus, the task required following the olfactory cue of the lemon scent on the target string; all other strings were unscented. Each trial began with the animal in an opaque start box. A guillotine door was opened to allow the animal to start out along the scented path. If the correct choice were made at the decision point and the animal followed the scented string to the target spot, it was allowed to eat the chocolate chip before being returned to the start box. If an incorrect choice were made at the decision point, if the animal ever left the correct string to follow another, or if the target were not reached within $30 \mathrm{sec}$, the animal was immediately returned to the start box. During training sessions, the direction of the scented string beyond the decision point was randomly varied such that each target was used an equal number of times.

Training. All animals were trained by a shaping procedure of increasing difficulty similar to that employed in Experiment 1 . Animals were first trained to follow the scented start string and then were gradually introduced to angular paths and choices between scented and unscented strings. The following stages were used: Level 1 -follow scented string to decision point $(55 \mathrm{~cm})$; Level 2-follow path to Target $4(110 \mathrm{~cm})$; Level 3follow path to Target 4 or Target 5 (one scented string); Level 4 Target 2, 4, 5, or 8 (one scented string); Level 5-Targets 1-8 (one scented string); Level 6-Targets 1, 3, 5, 7 (one scented, three unscented strings); Level 7-Targets 1-8 (one scented, seven unscented strings). At the first two levels of performance, criteria for advancing were identical to those used in Experiment 1. For Levels 3-8, eight trials were given each day, and animals were advanced to the next level if six of the trials were successfully completed.

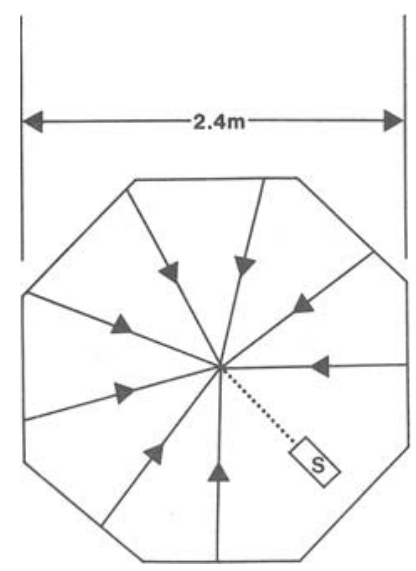

Figure 5. Diagram of chamber arranged for OTF testing. Dotted line indicates initial segment from start box (S) to decision point. Small triangles indicate target shields. 
Surgery. The animals were divided into three groups and operated on as in Experiment 1. Five animals received bilateral posterior caudate nucleus lesions, five animals received bilateral dorsal hippocampal lesions, and two animals were sham operated.

Retraining. Following the 10-day recovery period, the animals were started at the beginning of the training situation again. All animals were retrained to successful completion of Level 7.

Behavioral evaluation. For each trial (both before and after surgery), a record was made of the actual path followed by the rat. This allowed examination for directional biases and alternation tendencies, as well as records of successful performance. For this task, the basic measure of performance was taken to be the number of days required to master each level. From this individual measure, savings scores were again computed by subtracting postsurgical scores from presurgical scores.

Histology. Histological verification of lesion sites and size was carried out as in Experiment 1, with identical results.

\section{Results}

Directional biases. Examination of actual choices for evidence of perseveration and alternation showed little consistent bias interfering with performance. Perseveration was the most common error in early training, but later stages showed no consistent error pattern. A few animals showed left- or right-turn preferences, often related to the side of the string the animal chose to walk along. By Level 6, this tendency had disappeared. As in Experiment 1, no evidence of motivational effects was found in rates of refusal or percent-correct scores.

General performance. Increases in difficulty over levels had little effect on performance. Level 6, which required a choice between scented and unscented strings, showed an increase in difficulty, but Level 7, a direct variation of Level 6, was mastered very quickly.

Postsurgery scores showed that all animals progressed rapidly through the first five levels. Few animals required more than one session to reach criterion performance. As in the presurgery scores, Level 6 was more difficult for all groups, while Level 7 showed a general improvement in performance. Both experimental groups were consistently better after the surgery than before.

Savings scores. Analysis of savings scores (Figure 6) supported the observation that all groups showed improved performance following surgery. Savings scores for the three groups were compared using the Kruskal-Wallis rank-sum test (corrected for unequal groups). No significant between-groups difference was found $\left(\mathrm{H}^{1}=1.75, \mathrm{p}>.05\right)$. Since the sham-operated group was so small and was responsible for considerable postsurgical variability in the scores, an additional test of differences between the caudate and hippocampal groups was made. The Mann-Whitney U test showed no significant difference $(U=11, p>.05)$. Thus, caudate and hippocampal lesions did not differentially affect performance; both groups showed improved performance (positive savings), as would be expected in a test-retest paradigm.

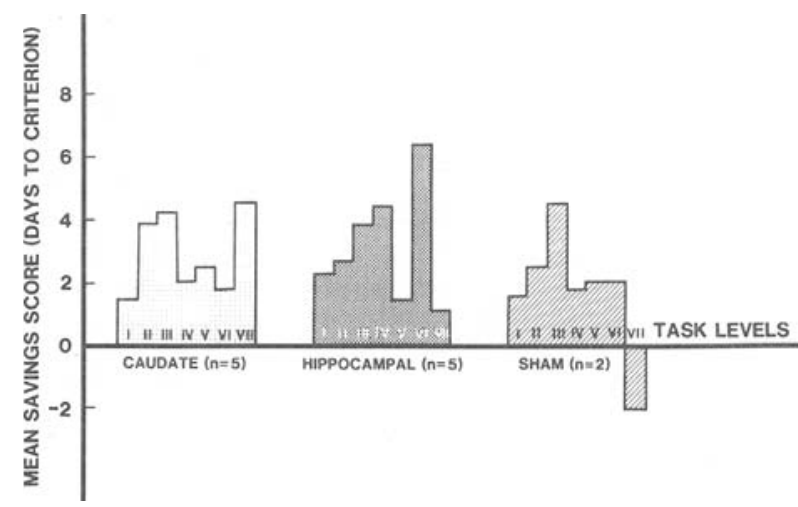

Figure 6. Experiment 2: OTF mean savings scores by task levels.

\section{Discussion}

Experiment 1 represented a test of egocentric spatial orientation behavior with an emphasis on proprioceptive cues (Beritoff, 1965). Replicating Miller, Barnett, and Potegal (Note 1), we were able to use appropriate strategies for shaping and motivating performance such that rats would return to a goal following passive transport away from the goal. This task required the animals to monitor the passive transport and then execute appropriate locomotion to return to the starting point. That is, the target had to be selected from among eight choices without the benefit of any exteroceptive cues. Our results indicated a specific deficit in RPT retraining for animals with bilateral posterior caudate lesions.

No evidence was found in Experiment 1 for hippocampal-lesion-related deficits in performance. This appears to be consistent with theories of hippocampal involvement in spatial memory and mapping for allocentric but not egocentric orientation (Jarrard, 1978; O'Keefe \& Nadel, 1978; Olton, 1977). Since only proprioceptive cues were available for the critical spatial memory and mapping that might be required, it is not surprising that the hippocampal animals showed no impairment when compared with the sham-operated controls. A similar lack of hippocampal-lesion effect in Experiment 2 is most easily explained by the fact that spatial memory per se was not required, since the relevant olfactory cues were present throughout the experiment to guide the animal's choice. However, before concluding that the hippocampus does not contribute to these spatial tasks, the effects of lesions larger than the ones used in these experiments as controls for the caudate lesions should be examined.

On the basis of the results from Experiment 2, we can conclude that neither caudate nor hippocampal lesions produced generalized deficits in spatial behavior to the extent that an olfactory trailfinding task was affected. Specifically, animals in both groups showed no differences in motivation or performance. 
This, in turn, supports the hypothesis that the posterior caudate nucleus is involved selectively in egocentric spatial orientation (Potegal, 1972). The use of the RPT task limited the available cues for encoding the target path to somatosensory and vestibular input. The caudate group was significantly impaired in relearning the task, whereas the hippocampal and sham groups showed positive savings. Since all three groups with similar lesions exhibited positive savings in Experiment 2, the involvement of the posterior caudate nucleus appears to be related to the specific nature of the spatial task. This is an extension of previous attempts to describe functional aspects of the posterior portion of the caudate (Livesey \& Muter, 1976; Studelska \& Beatty, 1978; Winocur, 1974).

The nature of vestibular involvement in egocentric spatial orientation remains unclear. Vestibular cues seem to play a large role in RPT (Miller, Potegal, \& Abraham, 1983). However, the caudate nucleus lesions did not appear to affect the labyrinthine righting reflexes as measured by head orientation at landing. Therefore, if the contribution of the caudate nucleus to egocentric spatial orientation involves vestibular influences, this mechanism appears to be separate from the vestibular influence on righting reflexes.

The results of Experiment 1 do indicate improvement over time for the caudate group, although never as much as before the lesions. This improvement in performance may have reflected the development of alternative strategies. For example, although Beritoff (1965), Miller, Potegal, and Abraham (1983), and Miller, Barnett, and Potegal (Note 1) showed that vision was not required for successful performance of RPT, it is conceivable that a combination of visual and somatosensory cues could provide enough information for some success in the early stages (short distances) by reducing the number of possible target alternatives.

In conclusion, these experiments support the hypothesis that the posterior caudate nucleus is involved in egocentric spatial orientation (Potegal, 1972). Furthermore, it appears that egocentric spatial orientation based on proprioceptive (vestibular) cues is not mediated by the hippocampus.

\section{REFERENCE NOTE}

1. Miller, S., Barnett, B., \& Potegal, M. Cues for path-finding with passive movement exposure to the path. Paper presented at the 49th annual meeting of the Eastern Psychological Association, Washington, D.C., April 1978.

\section{REFERENCES}

Beritoff, J. S. Neural mechanisms of higher vertebrate behavior. Boston: Little, Brown, 1965.

Copack, P. B., Dafny, N., \& Gilman, S. Neurophysiological evidence of vestibular projections to thalamus, basal ganglia, and cerebral cortex. In T. C. Frigyesi, E. Rinvik, \& M. Yahr (Eds.), Corticothalamic projections and sensorimotor activities. New York: Raven Press, 1972.

Douglas, R. Cues for spontaneous alternation. Journal of Comparative and Physiological Psychology, 1966, 62, 171-183.

Freeman, F. G. Cue utilization and hippocampal lesions in rats. Physiological Psychology, 1978, 6, 275-278.

Gross, C. G., Chorover, S. L., \& Cohen, S. M. Caudate, cortical, hippocampal and dorsal thalamic lesions in rats: Alternation and Hebb-Williams maze performance. Neuropsychologia, 1965, 3, 53-68.

JARRARD, L. E. Anatomical and behavioral analysis of hippocampal cell fields in rats. Journal of Comparative and Physiological Psychology, 1976, 90, 1035-1050.

JARRARD, L. E. Selective hippocampal lesions: Differential effects on performance by rats of a spatial task with preoperative versus postoperative training. Journal of Comparative and Physiological Psychology, 1978, 92, 1119-1127.

JuUrmaA, J., \& Suonio, K. The role of audition and motion in the spatial orientation of the blind and the sighted. Scandinavian Journal of Psychology, 1975, 16, 209-216.

KIRKBY, R. J. Caudate nucleus and arousal in the rat. Journal of Comparative and Physiological Psychology, 1973, 85, 82-96.

Livesey, P. J., \& Muter, V. Functional differentiation within the neostriatum of the rat using electrical (blocking) stimulation during discrimination learning. Journal of Comparative and Physiological Psychology, 1976, 90, 203-211.

Mikulas, W. L. Effects of lights at the choice point on spatial alternation and position learning by normal rats with bilateral lesions of the caudate nucleus. Psychonomic Science, 1966, 5, 275-276.

Miller, S., Potegal, M., \& Abraham, L. Vestibular involvement in a passive transport and return task. Physiological Psychology, 1983, 11, 1-10.

NeiLL, D. B., \& LiNN, C. L. Deficits in consummatory responses to regulatory challenges following basal ganglia lesions in rats. Physiology \& Behavior, 1975, 14, 617-624.

O'KeEfe, J., \& Dostrovsky, J. The hippocampus as a spatial map. Preliminary evidence from unit activity in the freely moving rat. Brain Research, 1971, 34, 171-175.

O'KeEFE, J., \& NADEL, L. The hippocampus as a cognitive map. Oxford: Oxford University Press, 1978.

Orton, D. S. Spatial memory. Scientific American, 1977, 236, 82-98.

Potegal, M. Role of the caudate nucleus in spatial orientation of rats. Journal of Comparative and Physiological Psychology, 1969, 69, 756-764.

Potegal, M. A note on spatial-motor deficits in patients with Huntington's disease: A test of a hypothesis. Neuropsychologia, 1971, 9, 233-235.

Potegal, M. The caudate nucleus egocentric localization system. Acta Neurobiologiae Experimentalis, 1972, 32, 479-494.

Potegal, M., Copack, P., de Jong, J. M. B. V., Krauthamer, G., \& Gilman, S. Vestibular input to the caudate nucleus. Experimental Neurology, 1971, 32, 448-465.

Potegal, M., Day, M. J., \& Abraham, L. Maze orientation, visual and vestibular cues in two-maze spontaneous alternation of rats. Physiological Psychology, 1977, 5, 414-420.

Ryugo, D. K., \& KillackeY, H. P. Differential telencephalic projections of the medial and ventral divisions of the medial geniculate body of the rat. Brain Research, 1974, 82, 173-177.

Schiff, B. B., \& Carter, D. Long-term effects of caudate nucleus lesions on body weight in the rat. Physiology \& Behavior, 1977, 18, 375-379.

Spiegel, E. A., Szekely, E. G., \& Gildenbera, P. L. Vestibular responses in midbrain, thalamus and basal ganglia. Archives of Neurology (Chicago), 1965, 12, 258-269.

Stoller, W. L. Food, water and saccharin solution intake in rats with posterior striatal lesions. Physiology \& Behavior, 1974, 13, 599-602. 
Studelska, D. R., \& Beatty, W. W. Open-field and avoidance behavior after neostriatal lesions in male and female rats. Journal of Comparative and Physiological Psychology, 1978, 92, 297-311.

Winocur, G. Functional dissociation within the caudate nucleus of rats. Journal of Comparative and Physiological Psychology, $1974,86,432-439$.

(Manuscript received October 21, 1982; revision accepted for publication February 14, 1983.) 